\title{
La formación de profesores en el estado de Sinaloa: I874-1984
}

\section{Teacher training in the state of Sinaloa: I874-1984}

\section{Dina Beltrán López*}

* Profesora-investigadora adscrita a la Universidad Autónoma de Sinaloa (México). Doctora en Educación Basada en Competencias por el Centro Escolar del Mar de Cortés (Culiacán, Sinaloa, México). Sus últimas publicaciones son el libro De la utopía a la realidad, de la realidad a la utopía. La universidad de Occidente en Sinaloa (1918-1922) (UAS, 2018) y el artículo "La profesión docente y las teorías implícitas sobre la enseñanza. Una reflexión crítica" (Qurriculum, 32, 2019, pp. 213-226). Es miembro del Sistema Nacional de Investigadores, nivel II. Su línea de investigación es Historia de la educación media y superior. Correo electrónico: dinabelt@uas.edu.mx

https://orcid.org/0000-0002-4988-4041

\section{Historial editorial}

Recibido: 06-abril-2020

Aceptado: 18-junio-2020

Publicado: 21-julio-2020

ISSN-e: 2594-2956 
La formación de profesores en el estado de Sinaloa: 1874-1984

\section{Resumen}

Con base en teóricos del currículo que consideran a este como un proceso históricamente condicionado y determinado por los factores del contexto, en este artículo se analizan los planes de estudio de la carrera de profesor normalista en el estado de Sinaloa, México, a lo largo de más de un siglo: desde que se instituyó la carrera de profesor de primeras letras en I874, en el antiguo Colegio Rosales, hasta I984, año en el que se elevó a nivel licenciatura. La investigación se realizó bajo el enfoque metodológico de la hermenéutica crítica, retomándose específicamente los planteamientos de Jürgen Habermas. Las instituciones educativas sinaloenses que se toman como base son la Escuela Normal que con distintos nombres dependió de las instituciones antecedentes de la hoy Universidad Autónoma de Sinaloa (de I88I hasta 1947), la Escuela Normal Urbana Vespertina que funcionó de 1947 a 1949 y la actual Escuela Normal de Sinaloa que inició sus funciones el primero de enero de 1950. En los resultados, se comparte una propuesta de periodización de la formación de los profesores en el estado de Sinaloa, la cual comprende cuatro etapas: a) equivalente a estudios primarios (I874-I895), b) equivalente a estudios de bachillerato (I895-1940), c) como subprofesión (1940I984) y d) como profesión (desde I984 hasta 202 la actualidad).

Palabras Clave: Currículo, Educación Normalista, Formación de Profesores. Planes de Estudio, Reforma Curricular.
Teacher training in the state of Sinaloa: I874-I984

\section{Abstract}

Based on theorists who consider curriculum a process historically conditioned and determined by contextual factors, this article analyzes the curricula of the Normalistic (primary school) teachers career in the state of Sinaloa, Mexico, over more than a century: from the beginning of the school teacher career at the old Colegio Rosales in 1874 , until 1984, when it became a bachelors' degree. This study was carried out under the methodological approach of critical hermeneutics, specifically taking up the approaches of Jürgen Habermas. The educational institutions of Sinaloa that ground the study are the Normal school which, under different names, depended on the previous institutions to what is now the Universidad Autónoma de Sinaloa (from I88I to 1947), the Escuela Normal Urbana Vespertina that operated from 1947 to 1949 , and the current Escuela Normal de Sinaloa which was founded on January I, I950. In the results section it is shared a proposal for the periodization of teacher training in Sinaloa, which is made of four stages: a) equivalent to primary studies (I874-I895), b) equivalent to high school studies (I895-I940), c) as a subprofession (1940-1984) and d) as a profession (from 1984 to the present).

Keywords: Curriculum, Curriculum Reform, Normal Education, Teacher Training, Primary school teachers. 
Formation des enseignants dans l'état de Sinaloa I874-1984

\section{Résumé}

Sur la base des théoriciens du curriculum qui le considèrent comme un processus historiquement conditionné et déterminé par des facteurs contextuels, cet article analyse les plans d'étude de la carrière d'enseignant normaliste dans les États de Sinaloa, au Mexique, à travers plus de un siècle: du début de sa carrière de professeur de lettres en I874 à l'Ancien Colegio Rosales, jusqu'en 1984, année où il accède au baccalauréat. La recherche a été menée dans le cadre de l'approche méthodologique de l'herméneutique critique, reprenant spécifiquement les approches de Jürgen Habermas. Les établissements d'enseignement antécédents de l'actuelle Université autonome de Sinaloa (de I88I à 1947), l'école normale du soir urbaine qui a fonctionné à partir de 1947), l'école normale du soir urbaine qui a fonctionné de 1947 à I949 et l'école normale actuelle de Sinaloa qui a commencé son fonctions le Ier janvier 1950. Dans les résultats, une proposition de périodisation de la formation des enseignants dans l'état de Sinaloa est partagée, qui comprend quatre étapes: a) équivalent aux études primaires (I874-I895), b) équivalent aux études secondaires (I8951940), c) en tant que sous-profession (19401984) et d) en tant que profession (de 1984 à nos jours).

Mots-clés: Curriculum, Enseignement normal, Formation des enseignants, Plans d'études, réforme des programmes.
Szkolenie nauczycieli w stanie Sinaloa: I874-I984

\section{Streszczenie}

$\mathrm{Na}$ podstawie teoretyków programu nauczania, którzy uważają to za historycznie uwarunkowany proces określony przez czynniki kontekstu, ten artykuł omawia programy nauczania na kierunku profesora normalisty w stanie Sinaloa, Meksyk, na przestrzenie stu lat: od ustanowienia kierunku nauczania poaczatkowego w roku I874 w byłym Colegio Rosales, aż do 1984 roku, w którym został podniesiony do poziomu licencjata. Metodologia badan to podejście krytyczne do hermeneutyki, w szczególności do J. Habermas. Instytucje oswiatowe $\mathrm{w}$ stanie Sinaloa w ramach ksztalcenia poaczatkowego oparte sa na szkole dla pedadgogow, która z różnymi nazwami zależała od Autonomicznego Uniwersytetu w Sinaloa (od I88I do I947), Popoludniowej Szkoly dla Pracujacych Nauczycieli, która działała od 1947 do 1949 i obecnej Szkoly Pedagogicznej Sinaloa, która rozpoczęła swoje funkcje w 1950 roku. W wynikach przedstawiona jest propozycja okresowania kształcenia nauczycieli w stanie Sinaloa, obejmująca cztery etapy: a) równoważne szkole podstawowym (I874I895), b) równoważne studiom w szkole średniej (I895-1940), c) jako szkoly policealnej (I940-1984) i d) i jako uniwersyteckiej (od I984 do chwili obecnej).

Slowa kluczowe: Program nauczania,oswiata, szkolenie nauczycieli,plany programów nauczania, reforma programu nauczania. 


\section{Introducción}

La profesión docente tiene un lugar especial en los estudios sobre educación y conocer su devenir a lo largo de la historia reviste importancia no sólo para comprender cómo se ha configurado el papel de los docentes en el pasado, sino para explicar y comprender las situaciones que condicionan en la actualidad la formación y el ejercicio de la actividad de estos profesionales, y para hacer proyecciones en el futuro. Agustín Escolano (I996) señala la necesidad de la reconstrucción histórica del oficio de maestro, al reconocer que este es un trabajo propedéutico "para definir su identidad cultural y profesional, para instalarse críticamente en una tradición y para avanzar con más rigor en la elevación de su condición" (Escolano, I996, p. 312).

En el presente artículo se analiza el currículo de la carrera de profesor normalista en Sinaloa (México) a lo largo once décadas: desde que se instituyó la carrera de profesor de primeras letras en I874 en el Colegio Rosales, hasta I984, año en que pasó de subprofesión a profesión al elevarse a nivel licenciatura. Las instituciones educativas sinaloenses que se toman como base son la Escuela Normal que con distintos nombres dependió de la institución rosalina ${ }^{\mathrm{I}}$ desde $\mathrm{I} 88 \mathrm{I}$ hasta 1947, la Escuela Normal Urbana Vespertina que funcionó de I947 a 1949 y la actual Escuela Normal de Sinaloa que abrió sus puertas el I de enero de I950, teniendo como sede todas ellas la ciudad de Culiacán, capital del estado de Sinaloa, México.

Para el análisis, nos apoyamos en los teóricos del currículo, quienes lo consideran un proceso históricamente condicionado y

204 determinado por los factores del contexto. Por un lado, acudimos a la aportación de Stephen Kemmis, quien visualiza al currículo como construcciones histórico-sociales, que deben ser estudiadas $\mathrm{y}$ comprendidas como tales, lo que significa que han de estudiarse con relación a las "condiciones históricas y sociales en que se producen sus diversas realizaciones concretas, prestando especial atención a la 
ordenación particular de su discurso" (Kemmis, I993, p. 44). Y, por otro lado, cobran pertinencia las propuestas de Sacristán y Pérez (2000), quienes en su conceptualización del currículo consideran, entre otras cosas, que se trata de un proyecto que sólo puede entenderse como un proceso históricamente condicionado, perteneciente a una sociedad, seleccionado de acuerdo con las fuerzas dominantes en ella, y con la capacidad no sólo de reproducir, sino también de incidir en la transformación de esa misma sociedad.

En cuanto al enfoque metodológico, se recurre a la hermenéutica crítica desarrollada por la Escuela de Frankfurt, en específico a la postura de Jürgen Habermas (I996), ya que la investigación se enfoca primordialmente en la interpretación de textos documentales. Este autor considera a la hermenéutica como el arte de entender el sentido lingüísticamente comunicable de los diversos textos consultados. Al enfrentarnos a dificultades de interpretación que se deben a la magnitud de la distancia cultural, temporal o social, se atiende especialmente la recomendación de que:

[...] podemos en principio señalar de qué informaciones adicionales tendríamos que disponer para poder entender: sabemos que hemos de empezar descifrando un alfabeto, familiarizándonos con un léxico o averiguando reglas de aplicaciones específicas del contexto. Dentro de los límites de tolerancia de la comunicación lingüística habitual podemos saber, en nuestro intento de esclarecer hermenéuticamente plexos de sentido ininteligibles, qué es lo que (aún) no sabemos (Habermas, 1996, p. 287).

Como técnicas de investigación hemos recurrido al análisis documental y al análisis de contenido. De acuerdo con Jaime Andréu, el análisis documental es "la operación, o conjunto de operaciones, tendentes a representar el contenido de un documento bajo una forma diferente de la suya original a fin de facilitar su consulta o localización en un estudio ulterior" (Andréu, 200I, p. 9). El análisis de contenido, por su lado, consiste en aquellas técnicas que explican y 
sistematizan "el contenido de los mensajes comunicativos de textos, sonidos e imágenes y la expresión de ese contenido con ayuda de indicios cuantificables o no (Andréu, 200I, pp. 3-4); con el objetivo de efectuar deducciones lógicas justificadas concernientes ya sea a la fuente o a sus efectos. La diferencia principal entre ambas técnicas es en su objetivo: mientras que para el análisis documental es representar de manera condensada la información para su almacenamiento y consulta, para el análisis de contenido es establecer inferencias o explicaciones en una realidad dada a través de los mensajes comunicativos.

La aplicación de las dos técnicas señaladas en la presente investigación se hizo de la siguiente manera. El análisis documental se realizó a partir del escrutinio de las fuentes documentales de primera y segunda mano en aras de lograr una reconstrucción de los planes de estudio que se aplicaron en los distintos momentos para, en seguida, hacer un cuadro clasificatorio de las diversas asignaturas que nos permitiera agruparlas y apreciarlas por bloques: área Científica, Ciencias Sociales y Humanidades, Lenguas, Área Práctica y Físico-Estética y Área Pedagógica. El análisis de contenido, por su parte, tuvo como propósito aportar explicaciones-comprensiones a ese cuadro clasificatorio de materias tratando de hacer inteligibles los sentidos y significados de los planes de estudio reconstruidos en los contextos específicos en que se pusieron en operación.

La formación de los docentes de educación primaria en el estado de Sinaloa constituye un campo que ha estado sujeto a diversas tensiones, de las cuales en este texto intentamos hacer visibles tres, a saber: a) la de desarrollarse como subprofesión o profesión; b) la de 206 seguir un derrotero propio a nivel estatal o ajustarse a los lineamientos nacionales; y c) el tipo de contenidos a incluir (prácticos, teóricos, humanísticos) y el peso de cada uno de ellos. El propósito en este artículo es, además de lo ya señalado, dar cuenta de estas tensiones en el periodo comprendido de I874 a I984. 


\section{Currículo equivalente a estudios de primaria (1874-1895)}

En I823 se fundó en la capital del país la Escuela Normal Lancasteriana en la que se instituyó el método de enseñanza del mismo nombre, que consistía en preparar como monitores a los niños más avanzados para que estos, a su vez, se encargara de enseñar a grupos de diez o veinte niños (Curiel, 20I0). En Sinaloa dicho método se estableció 46 años después, cuando en julio de i869 el Congreso del Estado facultó para otorgar títulos de preceptores de primeras letras a la escuela primaria para niños pobres que la Compañía Lancasteriana había establecido en el puerto de Mazatlán, Sinaloa, (Beltrán y Berrelleza, I998).

En el plan de estudios para el estado de Sinaloa aprobado en i874 que crea el Colegio Rosales, se estableció que para ser profesor de primeras letras se requerían todos los conocimientos de la educación primaria y tener práctica en enseñanza (Periódico Oficial del Estado de Sinaloa [POES], 25 de marzo, I874). Este plan de estudios contenía las mismas materias que la instrucción primaria de segunda clase, las cuales se podían cursar en un año; pero con una precisión: cosmografía era la única asignatura en que se diferenciaban los estudios normalistas de los primarios (Sánchez, 2000).

En I88I la Junta Directiva de Estudios del estado de Sinaloa acuerda crear la Escuela Normal de Preceptores dependiente del Colegio (POES, 2I de mayo, I88I) y dos meses después se aprobó su reglamento que contiene el plan de estudios de la carrera de maestro y en el que se indica que se cursaría en dos años (POES, I3 de agosto, I88I). Salvo que contempla un curso de Pedagogía, los estudios de normal seguían teniendo más cercanía con el currículo de la educación primaria que con el de preparatoria; contenía un total de I7 cursos. 
Contrasta este plan de estudios sinaloense con los que se pusieron en marcha años después en las escuelas normales de Jalapa (I886) y de México (I887) que tenían 47 y 43 cursos para estudiarse en 5 y 4 años, respectivamente. Según se desprende del análisis, en dichas escuelas varias de las materias eran del campo de la enseñanza normalista tales como: Pedagogía con elementos de psicología, Metodología con especialidad en el sistema Fröebel, Caligrafía y dibujo aplicado a la enseñanza, Canto coral, Nociones de medicina doméstica y de higiene doméstica y escolar con práctica de la vacuna, Organización y disciplina escolar, e Historia de la pedagogía (Meneses, I998a).

\section{Currículo equivalente a estudios de bachillerato}

En pleno porfiriato, bajo el gobierno del general Francisco Cañedo, en I895 se aprobó una nueva ley de instrucción pública para Sinaloa que reformó los planes de estudio del Colegio Rosales. La carrera normalista se amplió de dos a cuatro años y el número de cursos aumentó de 17 a 27 (POES, I y 7 de marzo, I885). En lo relativo a las asignaturas propias de la educación normalista, se incluyen solamente dos cursos de Pedagogía y uno de Higiene escolar.

Puede considerarse que con este plan de estudios se da un salto cualitativo significativo ya que su contenido se acerca más a los estudios preparatorios que a los de la primaria, pero con diferencias sustantivas con relación al recomendado a nivel nacional. De acuerdo con Meneses (I998a), en el Segundo Congreso Nacional de Instrucción Pública (I89I-I892) se orientó a que cada entidad

208 federativa tuviese una escuela normal dividida en dos niveles, uno para primaria elemental y otro para primaria superior, y que hubiera una para hombres y otra para mujeres; recomendaciones que Sinaloa no retomó. 
Durante el desarrollo del proceso revolucionario, en noviembre de 19I5, el Congreso local nombró gobernador provisional de Sinaloa al ingeniero Manuel Rodríguez Gutiérrez, quien promovió diversos cambios en el ámbito educativo que incluyeron una reforma del plan de estudios de la carrera magisterial que se impartía en el Colegio Rosales. Dentro de los cambios contemplados, se extendió la duración de la carrera de 4 a 5 años y las materias aumentaron de manera significativa con relación al plan de i895, pues tenía un total de 53 (POES, 27 de enero de I9I6). No obstante, la modificación más importante fue que se incluyó una cantidad considerable de cursos orientados a la preparación práctica para el ejercicio docente, pero con una escasez de los orientados a la formación pedagógica. También es pertinente subrayar que, aunque no estén delimitados de manera explícita los ciclos secundario y profesional, sí lo están implícitamente, pues la mayoría de los cursos para formar en la docencia se concentran en cuarto y quinto años, mientras que en los tres primeros años escolares se concentran las materias relacionadas con el plan de estudios de preparatoria, nivel educativo que también fue objeto de reforma. Como conclusión, puede considerarse que este plan de estudios representó un intento de enriquecer la parte práctica de la formación docente, es decir, sobre el cómo enseñar.

Sin embargo, como las condiciones del contexto socioeconómico y político eran difíciles, el anterior plan de estudios no prosperó, entre otras cosas porque implicaba una mayor erogación por concepto de sueldo a los profesores, además de que, al estar el Colegio bajo la tutela del gobierno del estado, sus autoridades no eran estables al no serlo tampoco quienes ocupaban la gubernatura (Beltrán, 2015a). Para dar idea de la inestabilidad reinante en los gobiernos de estos años, basta decir que desde la muerte del gobernador general Francisco Cañedo, ocurrida en junio 1909, y hasta mediados de 1917, Sinaloa había tenido 28 gobernadores: 8 constitucionales, I3 interinos, 5 provisionales, un sustituto, $y$ un gobernador $y$ comandante militar (Berrelleza, I998). 
Finalizada la fase armada de la Revolución mexicana, en I9I7 se realizaron en Sinaloa las elecciones constitucionales para gobernador, en las que triunfó el general Ramón F. Iturbe, dejando en el camino las aspiraciones del general Ángel Flores, quien fue su principal contrincante. Iturbe llegó con diversos proyectos para mejorar la educación y, para lo concerniente a la normalista, impulsó dos propuestas. La primera fue la aprobación del Plan de Estudios para la Carrera de Maestro de Enseñanza Elemental, que tenía como propósito dotar de más herramientas y conocimientos a los docentes en servicio (POES, I de junio, I9I8); y la segunda se enfocó en la renovación curricular de la carrera de profesor normalista del Colegio Rosales. En el primer año de su mandato, este último fue transformado en una universidad regional dotada de régimen de autonomía a la que se denominó Universidad de Occidente.

Los cambios curriculares de la carrera magisterial en la Universidad, con respecto al aprobado para el Colegio Rosales en I895, fueron significativos, pero se conservó la duración de cuatro años. En el aspecto cuantitativo el número de materias pasó de 23 a 3I, y en el cualitativo, se incluyeron cinco materias nuevas propias del campo de la educación normalista: Pedagogía general, Labores domésticas, Práctica de observación, Metodología del canto y Trabajos manuales. Excepto para Trabajos manuales que incluyó tres cursos, para las otras cuatro materias era sólo uno (Beltrán, 20I8).

El hecho de que de las 3I materias del plan de estudios de la carrera de profesor normalista (tenía esta denominación desde 1917) 24 coincidan con las del plan del bachillerato de la Universidad de Occidente, permite concluir que se mantenía y acentuaba la

210 tendencia de hacer coincidir o hermanar los currículos de estos dos niveles educativos.

Además de las modificaciones en el mapa curricular, un aspecto que sobresale es que, al iniciar la Universidad, se cambió el requisito de ingreso a las diversas carreras (incluida la de profesor normalista) con 
respecto al nivel de estudios primarios requeridos. Mientras que la Ley de Instrucción Pública de 1895 establecía que sólo se requería haber concluido la primaria elemental, la nueva legislación ordenó que el alumno debía haber terminado la primaria superior, lo cual representó la elevación del nivel de los estudios normalistas (Beltrán, 20I5a).

El proyecto de la Universidad de Occidente fracasó porque no se le proveyeron los recursos necesarios que aseguraran su adecuada marcha, como resultado del enfrentamiento entre los miembros de la clase política (Beltrán, 20I5a). Ocurrió que, durante el mandato de Iturbe, el candidato a quien derrotó en las urnas —el general Ángel Flores - nunca asimiló haber perdido la gubernatura y, por ello, se dedicó a obstaculizar los diversos proyectos, conflicto que tuvo como telón de fondo el enfrentamiento entre el presidente Carranza y el grupo de los sonorenses del que formaba parte el general Álvaro Obregón (Beltrán, 2018).

Clausurada la Universidad de Occidente, en octubre de I922, se aprobó la creación del Colegio Civil Rosales, institución que conservó la facultad de impartir los estudios normalistas (POES, 26 de octubre, I922). Si bien en un primer momento se estableció que para ingresar a ella se requerían sólo los estudios primarios elementales, fue un requisito que enseguida se corrigió restableciéndose que era menester haber concluido los de primaria superior (POES, I9 de diciembre, 1922).

Un cambio importante fue que la duración de la carrera se redujo de cuatro a tres años y el número de asignaturas de 3I a 25. Hubo modificaciones en las materias propias de la carrera de profesor normalista y algunos aspectos del nuevo currículo muestran claramente que la intención era restablecer los rasgos de la formación magisterial del antiguo Colegio Rosales. El punto más significativo era que se procuraba ahorrar en el pago de la nómina de los profesores pues al reducir el número de asignaturas la erogación por conceptos de salarios disminuiría. 
$\mathrm{Al}$ influjo de los vientos reformadores latinoamericanos iniciados en la Universidad de Córdoba, Argentina, desde 1923 la Federación Nacional de Estudiantes Universitarios Mexicanos empezó a realizar una serie de congresos nacionales para demandar, entre otras cosas, la autonomía universitaria. Los estudiantes rosalinos, incluidos los normalistas, se incorporaron a este movimiento como Federación de Estudiantes Rosalinos (Beltrán, 2007) y en octubre de I93I propusieron la refundación del Colegio que, al aprobarse, entre otras cosas se expresó en la inclusión de los estudiantes en los órganos de gobierno institucional, no sólo representando a sus escuelas, sino también como organización (POES, 23 de octubre de I93I).

Todo lo anterior significó cambios curriculares en las distintas carreras y la de profesor normalista no fue la excepción. En esta última se incrementaron las materias (de 25 a 34), se eliminaron otras, se empobrecieron los ejes de la formación científica y el de Ciencias Sociales y Humanidades, y las áreas que agrupaban las materias prácticas y de carácter físico-estético se reestructuraron. Es oportuno subrayar que el área pedagógica pasó de 3 a 7 materias, observándose con las nuevas, que la Pedagogía estaba cediendo espacio a unas ciencias de la educación cada vez más diversificadas. Puede concluirse que con este plan de estudios se dio prioridad a los contenidos enfocados a que el alumno de esta carrera aprendiera a hacer cosas sobre los de carácter teórico-conceptual (Beltrán, 20I6).

El anterior plan de estudios se reformó en enero de 1933 porque pronto afloraron las deficiencias producto del desequilibrio en los diversos tipos de contenidos, además de que no fue fácil sostener el pago a profesores de 34 materias, en una situación en que la crisis

212 económica era un problema cotidiano (Beltrán, 2007). El propósito de esta reforma fue lograr un mayor equilibrio entre los contenidos teóricos y prácticos, por lo que se incluyeron cursos como Español práctico, Historia de México, Sociología y Economía. 
A los vientos reformistas provenientes del Cono Sur latinoamericano en favor de la autonomía universitaria, que soplaban fuertemente en el país, se sumaron los de la reforma educativa socialista que se concretó en la reforma del Artículo $3^{\circ}$ Constitucional la cual empezó a aplicarse en la presidencia del general Lázaro Cárdenas (I934-I940) (Beltrán, 2007).

En Sinaloa, esta política educativa impactó de manera significativa al grado de que el máximo órgano de gobierno acordó, en octubre de 1934, hacerla suya (AHUAS. [Archivo Histórico de la Universidad Autónoma de Sinaloa], 1930) y, dos años más tarde, se expresó en un cambio de mayor trascendencia: la transformación del Colegio Civil Rosales en la Universidad Socialista del Noroeste (POES, 27 de febrero, 1937). En esta reforma institucional en la que de nuevo se dio el tránsito de Colegio a Universidad, tuvieron amplia participación diversas organizaciones estudiantiles y magisteriales, y también el Partido Comunista, que formuló proyectos en los que plasmaron sus ideas sobre el ser y deber ser del centro de estudios (Beltrán, 2007).

Con el trasfondo del debate nacional y estatal sobre la educación socialista, dos reformas curriculares más se aprobaron en la década de I930, una en I935 y otra en I939. Aunque en el currículo de I935 se hicieron pocos cambios, estos fueron de relevancia porque se introdujeron dos asignaturas que daban el retoque socialista que impregnaba el ambiente de la época como son Historia de las doctrinas y luchas sociales, y Legislación revolucionaria. También se incorporó la materia de Economía política y problemas económicos de México, se restituyó el curso de Sociología aplicada a la educación, presente en el currículo de I93I, y al curso de Higiene escolar se le agregó el término "y social”.

En el plan de estudios aprobado en 1939, el "tinte socializante" se acentuó, lo que subrayamos porque estaba por concluir el sexenio cardenista y la reforma educativa continuaba sin afianzarse. Los cambios más relevantes en el citado plan fueron el aumento del número de asignaturas de 30 a 33 y la reestructuración de los ejes de 
Ciencias Sociales y humanidades, y el pedagógico. En el primero desapareció la asignatura de Historia de las doctrinas y luchas sociales y, en su lugar, se introdujo la de Teoría del cooperativismo; la de Literatura general fue sustituida por la de Historia crítica del arte y la literatura; se incorporó como novedad un curso de Psicología general; y a las materias relacionadas con la Historia ahora se les llamó Historia de la civilización. En lo que al eje pedagógico se refiere, desaparecieron los dos cursos de Psicología de la educación y los dos de Principios de educación, y se incorporaron tres cursos de Técnicas de la enseñanza, dos de Ciencias de la educación, uno de Paidología y otro de Psicotécnica pedagógica, por lo que este eje pasó de 7 a Io cursos. Al suprimirse la materia de Español práctico el eje de lenguas quedó vacío y el del área científica se mantuvo, pero sólo con el curso de Biología (Beltrán, 2016).

\section{La carrera de maestro nace como subprofesión}

El I8 y i9 septiembre de I940 el Consejo Universitario aprobó el reglamento general de la institución en el que ya no se reivindican los principios de la educación socialista. En lo que concierne a la enseñanza normal, se establece de manera escueta que se desarrollaría en un ciclo secundario y otro profesional, con tres años de duración cada uno (AHUAS, 1939). Subrayamos esto porque es una propuesta que no sólo puso freno, sino que dio reversa al proceso de empobrecimiento que había venido sufriendo el currículo de la carrera magisterial y es el documento legal que marca el inicio de la carrera de profesor normalista como subprofesión. En la Escuela

214 Nacional de Maestros la división de los estudios en dos ciclos ocurrió en I924, como resultado de la diferenciación que se hizo en i923 de los estudios preparatorios (Meneses, I998b).

El primero de octubre de I94I la Universidad Socialista del Noroeste se transformó a Universidad de Sinaloa (POES, I8 de octubre, I94I) y 
dos días después el Consejo Universitario aprobó los nuevos planes de estudio para las diversas carreras. Para la de profesor normalista, el plan incluyó 57 materias organizadas en un ciclo secundario (29 cursos) y otro profesional (28 cursos), observándose en las del ciclo profesional un equilibrio entre los contenidos de formación general, de formación técnica y de formación teórica en el campo pedagógico (Beltrán, 20I6). La desaparición de las materias socialistas muestra que, de manera temprana, el estado de Sinaloa definió su postura en este polémico tema del debate nacional.

En enero de 1945 empezó a gobernar en Sinaloa el general Pablo Macías Valenzuela y una de las primeras medidas fue expedir una nueva ley de educación para la entidad porque la que se tenía era obsoleta, pues databa de i9I7. Las condiciones en la entidad habían cambiado y era necesaria una nueva norma jurídica que, además de regular lo relativo a la educación primaria, incluyera todos aquellos niveles y tipos de educación que eran una realidad en el terruño sinaloense (Beltrán, 20I5b; Beltrán, 20I6).

En este instrumento legal se sentaron las bases para que el gobierno de Sinaloa asumiera la dirección de la educación normalista que hasta entonces había estado a cargo de la institución rosalina. Para concretar esto último, en abril de 1947 el Congreso del Estado aprobó la creación de la Escuela Normal Urbana Vespertina a partir del ciclo escolar I947-I948, mandatándose la supresión de los tres años del ciclo profesional de la Escuela Normal dependiente de la Universidad de Sinaloa (Beltrán y Berrelleza, I998; Beltrán 20I5b; Beltrán, 20I6).

El plan de estudios aprobado tenía 77 cursos anuales, 40 para el ciclo secundario y 37 para el profesional. De manera general, la observación es que en el ciclo secundario se da preferencia a la formación en el ámbito de las Ciencias Sociales y de las actividades, en tanto que en el profesional las de mayor peso son las materias de formación general y las de actividades. Asimismo, no se incluyeron las asignaturas reconocidas como "socializantes", lo que constituyó la 
diferencia fundamental con relación al plan que se puso en marcha en la Escuela Nacional de Maestros en 1945. Este cambio era pertinente porque, después de un largo y enconado debate, con la reforma del Artículo $3^{\circ}$ de 1946, se concretó la eliminación de la educación socialista y se dio paso a la etapa de la unidad nacional (Beltrán, 20I6).

El plan de estudios implementado en la Escuela Normal Urbana Vespertina a partir de 1947 -y continuado en la Escuela Normal de Sinaloa- se mantuvo hasta I960, ya que, durante la presidencia del licenciado Adolfo López Mateos, en el marco del Plan de Once años para la Expansión y Mejoramiento de la Educación (I959-1970), en noviembre de 1960 se realizó la Segunda Asamblea Nacional Plenaria del Consejo Nacional Técnico de la Educación, con el propósito de discutir un nuevo currículo para la educación normalista del país.

En la citada Asamblea se esbozó un perfil para el maestro y se emitió un nuevo plan de estudios con dos etapas: una cultural de un año y otra profesional de dos, organizado semestralmente, en la que cada semestre debía contemplar un máximo de tres materias. Asimismo, se le dio cierta flexibilidad al currículo al incluir asignaturas opcionales, lo que tenía la intención de atender las necesidades de los alumnos y las características regionales (Meneses, 1998c). Excepto el lineamiento de la organización semestral de las materias, las disposiciones del Consejo Nacional Técnico de la Educación fueron retomadas en Sinaloa, con la aclaración de que en esta entidad federativa aún se incluía la educación secundaria.

Hacia finales de la década de I960, la carrera de profesor normalista 216 de la Escuela Normal de Sinaloa fue objeto de una reforma local. En I968, durante la gestión directiva del profesor Antonio Zazueta Armenta, con la asesoría de la Escuela Normal Veracruzana, se impulsó un cambio curricular que introdujo elementos novedosos. El resultado fue un plan equilibrado en las distintas áreas formativas (Beltrán y Berrelleza, 1998; Beltrán, 20I5b; Beltrán, 2016). 
Esta reforma, que implicó la separación gradual de la escuela secundaria de la Escuela Normal de Sinaloa, comenzó a implementarse en ese año. Aunque este plan de estudios sólo se aplicó durante un ciclo escolar, tiene relevancia porque significó adelantarse a los cambios que se harían enseguida a nivel nacional, además de que fue una experiencia local que muestra que el control del currículo de las escuelas normales del país por parte del Estado mexicano todavía no era total. Se trata de la última reforma curricular en cuya formulación las autoridades educativas de Sinaloa tuvieron un importante grado de autonomía.

Entre abril-mayo de 1969 se realizó en la ciudad de Saltillo, Coahuila, el Cuarto Congreso Nacional de Educación Normal. En julio siguiente el Consejo Nacional Técnico de la Educación ratificó los acuerdos de dicho Congreso y dictaminó, entre otras cosas, iniciar la separación de la secundaria en las escuelas normales que aún la conservaban, ampliar el ciclo profesional a cuatro años, adoptar formas para revalorar al maestro de primaria y aplicar las recomendaciones en el campo de la formación de maestros de educación preescolar (Meneses, 199I).

Las reformas curriculares que vinieron después no tienen mayor complicación pues son simples adaptaciones de los programas emanados de las diferentes reuniones nacionales. El currículo propuesto por el Congreso de Saltillo tuvo una corta vigencia ya que en 1972 la Asamblea Nacional de Educación Normal lo reformó, aunque los cambios no fueron substanciales, ya que lo que se hizo fue agrupar las materias por bloques (formación científica, humanística, psicopedagógica, tecnológica y la física y artística), además de que no incluyó asignaturas optativas que sí estaban contempladas en el plan de 1969.

Sin embargo, en 1975 se realiza una nueva reforma curricular en la que, aparte de que se cambia buena parte de las materias, muchas de ellas se asocian a la didáctica, demostrándose con ello la preocupación excesiva por los aspectos técnicos de la formación 
docente, en detrimento de una formación integral. Debido a que enseguida se advirtió lo inadecuado e inoperante de estos cambios curriculares, de inmediato fueron corregidos y de este proceso resultó el plan de estudios 1975 reestructurado (Curiel, 20IO). La diferencia en el eje pedagógico de uno y otro plan es que mientras el primero contemplaba cuatro cursos de Historia de la educación, el segundo tenía dos, más uno de Pedagogía general y otro de Didáctica general.

Las tres últimas reformas curriculares (la de 1972 y las dos de 1975) fueron aplicadas prácticamente sin cambios en Sinaloa, con lo que se puso en evidencia la tendencia a centralizar cada vez más en las autoridades educativas federales el diseño del currículo de la educación normal, reduciendo con ello el papel de los profesores al de simples operarios o técnicos.

\section{De subprofesión a profesión: la reforma de 1984}

La reforma curricular que dio la pauta para que las carreras de profesor normalista y de educadora de párvulos ${ }^{2}$ se elevaran a rango de licenciatura fue producto de un acuerdo entre la presidencia de Miguel de la Madrid Hurtado (I982-I988) y el Sindicato Nacional de Trabajadores de la Educación. En la década precedente el país había experimentado un acelerado crecimiento de la matrícula en educación superior y en los dos sexenios presidenciales anteriores el de Luis Echeverría Álvarez (I970-1976) y el de José López Portillo (1976-1982) — se habían aplicado una serie de políticas que propiciaron una profunda crisis económica, que fue enfrentada por

218 de la Madrid implementando un conjunto de medidas orientadas a la racionalización de la administración pública, la instauración del modelo neoliberal (el desmantelamiento del Estado benefactor e impulso de las leyes del libre mercado) y el inicio de un proceso de ordenamiento que se acompañó de transformaciones institucionales, organizativas y curriculares (Ducoing, 2013). 
En este contexto y en respuesta a una demanda del Sindicato Nacional de Trabajadores de la Educación, avalada también por la disidencia sindical, el presidente de México (I982-I988) puso en marcha una reforma curricular a nivel nacional que marcó un punto de inflexión en el campo de la formación de maestros en México, ocupación que desde su origen se desarrolló bajo el estigma de poseer un estatus menor al resto de las profesiones (Rosales, 2009). El proceso de profesionalización se había iniciado en 1975 con base en el Acuerdo II 298 de la Secretaría de Educación Pública, publicado en el Diario Oficial de la Federación, donde se estableció que todo egresado de las escuelas normales debía poder transitar a otros niveles y grados académicos del subsistema de educación superior; y se fortaleció con la creación de la Universidad Pedagógica Nacional en 1978 (Ducoing, 20I3).

Con la reforma curricular de 1984 nacieron las licenciaturas en educación primaria y en educación preescolar, estableciéndose que para poder ingresar a ellas era requisito haber concluido el bachillerato. Se consignó que la duración de ambas sería de cuatro años con una distribución semestral de las asignaturas (Rosales, 2009). La demanda sindical de homologar a profesión estas carreras, se relacionó con la mejora de las condiciones salariales y laborales, como una consecuencia natural del aumento de los años de escolaridad (Ducoing, 20I3).

Este modelo curricular posee varias diferencias respecto al plan de estudios de 1975 restructurado, entre las que destacan: a) Se redujo la carga curricular, pues de 75 cursos pasó a tener 6o, al eliminarse aquellos relacionados con los estudios de bachillerato; b) La propuesta curricular se estructuró con base en un tronco común para ambas licenciaturas y un área de formación específica correspondiente al nivel en el que se ejercería la docencia. El tronco común se conformó con las llamadas materias instrumentales y otro conjunto de asignaturas agrupadas en las líneas formativas social, pedagógica y psicológica; c) Se incorpora con un peso significativo la formación en investigación educativa; y e) Se suprimen los cursos de 
didáctica y didáctica especial, introduciéndose en su lugar las asignaturas denominadas Laboratorio de Docencia presentes en el currículo a partir del tercer semestre y se incorporan, entre otros, los cursos de Teoría educativa, Diseño curricular, Planeación educativa, Problemas de aprendizaje, Evaluación y Pedagogía comparada (Rosales, 2009; Ducoing, 2013).

Diversas son las críticas que se le han hecho al plan de estudios de I984 de parte de los investigadores educativos del campo curricular. En el caso de Sinaloa, la falla más importante que se le ha señalado es que a los docentes de las escuelas normales les faltó dominio y conocimiento de dicho plan, así como profundizar en su análisis para entenderlo y darle el sentido que concibieron quienes lo elaboraron (Rosales, 2009).

\section{Reflexiones finales}

En este recorrido se analizaron los currículos implementados en la carrera magisterial en Sinaloa a lo largo de once décadas, mostrando la evolución que siguió el proceso de formación de maestros hasta alcanzar el estatus de profesión en 1984. A lo largo del texto queda manifiesto que, hasta finalizar la década de 1960, las autoridades educativas de Sinaloa tuvieron e hicieron uso de un importante grado de autonomía en la elaboración de sus planes de estudios, la cual perdieron de manera significativa en la siguiente década y de manera total con la reforma de 1984 .

220 A partir del análisis de los contenidos en cada uno de los currículos que se pusieron en marcha en Sinaloa, quedan delineadas maneras distintas de concebir al profesor sea como: a) un sujeto que aprende a hacer cosas; b) un profesional de la docencia que además de apropiarse contenidos prácticos, adquiere un conjunto de conocimientos teóricos que acrecientan su capital cultural y le 
permiten desarrollar los procesos de reflexión para analizar de manera crítica su entorno e intervenir en él con un actitud transformadora; y/o c) un formador que posee herramientas teóricas y metodológicas para investigar con perspectiva científica en su campo. De la presencia/ausencia de estos tipos de contenidos, y la prioridad que se les dio, ha sido la visión del docente y de la escuela plasmada en cada documento curricular.

Aunque para el sentido común lo anterior carece de relevancia, para los estudiosos de estos temas es un asunto que preocupa porque precisamente en la tarea de definir y decidir respecto a qué contenidos se le da más importancia, es donde radica la principal tensión a resolver en el momento de diseñar y poner en operación cualquier currículo. Y desde luego que ello manifiesta una tensión más general respecto a los fines últimos de la educación: educar para reproducir o educar para transformar.

Sin duda que un problema nodal que se enfrenta cuando se diseña y pone en operación un programa curricular es sobre qué y cómo hacer para que el documento formal corresponda lo más posible con su aplicación en la realidad, es decir, de qué manera acortar la distancia entre el currículo formal y el real u operacional (Posner, 1998). Consideramos que en este tema radica el punto neurálgico a resolver y ahí es donde conviene tener presente que ningún currículo se aplica desligado de su entorno; por el contrario, como lo afirma Kemmis (1993), los factores del contexto ejercen una influencia fundamental que debe tenerse muy presente.

En los momentos actuales en que la educación mexicana atraviesa por una profunda crisis, urge volver nuestra mirada hacia nuestras instituciones formadoras de docentes, en tanto centros educativos con un papel esencial en la formación de los ciudadanos que se necesitan para construir un México más justo. El propósito deberá ser realizar un diagnóstico lo más objetivo posible para, a partir de él, plantear estrategias orientadas a mejorarlas, considerando que, si bien estas instituciones tienen problemas comunes, poseen también 
particularidades que a menudo se ignoran en los estudios nacionales. Las lecciones que aporta la historia en esta tarea han sido y son de un valor inigualable y todavía más en esta era en que la incertidumbre y la complejidad se acrecientan.

\section{Referencias}

AHUAS. [Archivo Histórico de la Universidad Autónoma de Sinaloa]. (1930). Libro de actas del Consejo Directivo, I930-I936 [Fondo Colegio Civil Rosales]. Sinaloa, México.

AHUAS. (1939). Libro de actas del Consejo Universitario, I939-I94I [Fondo Universidad Socialista del Noroeste]. Sinaloa, México. Andréu Abela, J. (200I). Las técnicas de análisis de contenido: Una revisión actualizada. Recuperado de: http://mastor.cl/blog/wp-content/uploads/2018/o2/Andreu.analisis-de-contenido.-34-pags-pdf.pdf

Beltrán López, D. (2007). La autonomía universitaria en la institución rosalina: I872-2006 [Tesis de licenciatura]. Centro de Investigaciones y Servicios Educativos de la UAS, Culiacán, Sinaloa, México.

Beltrán López, D. (20I5a). Currículum y educación media superior en la institución rosalina. Un estudio desde la historia cultural [Tesis del Doctorado de Educación Basada en Competencias]. Centro Escolar del Mar de Cortés, Culiacán, Sinaloa, México.

222 Beltrán López, D. (20I5b). La formación de maestros en Sinaloa: la Escuela Normal de Sinaloa y otras instituciones formadoras de docentes (1945-1980). En M.Á. Rosales Medrano, D. Beltrán López y J.L. Sánchez Gastélum (Coords.), Educación y Política, Tomo VI, Colección Historia Temática de Sinaloa (pp. 235-27I). 
México: Gobierno del Estado de Sinaloa / Instituto Sinaloense de Cultura / Consejo Nacional para la Cultura y las Artes.

Beltrán López, D. (2016). Currículum y educación normalista en Sinaloa, I9I7-I980. En P. Moreno, M. de J. Soto, y J. J. Castro (coords.), El Sistema Educativo en Sinaloa. Políticas curriculares y temas emergentes (tomo II) (pp. 97-I36). México: Universidad Pedagógica del Estado de Sinaloa / Ediciones del Lirio.

Beltrán López, D. (20I8). De la utopía a la realidad, de la realidad a la utopía. La Universidad de Occidente en Sinaloa (I9I8-1922). México: Universidad Autónoma de Sinaloa.

Beltrán López, D., y Berrelleza Fonseca, M. A. (1998). Escuela Normal de Sinaloa. Una visión histórica. México: Escuela Normal de Sinaloa.

Berrelleza, M. A. (1998). De Liceo a Universidad. La institución rosalina: I872-I922. Culiacán, Sinaloa: Universidad Autónoma de Sinaloa.

Curiel Méndez, M.E. (20IO). La educación Normal. En F. Solana, R. Cardiel Reyes y R. Bolaños Martínez, (Coords.) Historia de la educación pública en México (I876-1976) (pp. 426-462). México: Fondo de Cultura Económica.

Ducoing, P. (Coord.) (2013). De la formación técnica a la formación profesional: la reforma de la educación normal de i984. En P. Ducoing, La Escuela Normal. Una mirada desde el otro (pp. II7I56). México: UNAM-IISUE. Recuperado de: http://I32.248.192.24I/ editorial/wpcontent/uploads/20I4/Io/La-Escuela-Normal.pdf

Escolano Benito, A. (1996). Memoria de la formación de maestros

(Conferencia pronunciada en la conmemoración de los 150 años de la Escuela de magisterio de Zamora). Aula, 8, 3II-327. Recuperado

de: 
http://campus.usal.es/ revistas_trabajo/index.php/o2I43402/article/viewFile/3459/3479

Gimeno Sacristán, J., y Pérez Gómez, A. (2000). Comprender y transformar la enseñanza. Madrid: Ediciones Morata.

Habermas, J. (1996). La lógica de las Ciencias Sociales. Madrid: Tecnos.

Kemmis, S. (1993). El curriculum: más allá de la teoría de la reproducción.

Madrid: Ediciones Morata.

Márquez Carrillo, J. (2015). Universidad, Modernización y Reforma en Puebla. La estela de tres proyectos rectorales, I948-1965. Graffylia. Revista de la Facultad de Filosofía y Letras, (20), 5-I6. Recuperado de:

http://cmas.siu.buap.mx/portal_pprd/work/sites/filosofia/res ources/PDFContent/1316/002.pdf

Meneses Morales, E. (I99I). Tendencias educativas oficiales en México, 1964-I976. México: Centro de Estudios Educativos / Universidad Iberoamericana.

Meneses Morales, E. (I998a). Tendencias educativas oficiales en México, I82I-I9II. México: Centro de Estudios Educativos / Universidad Iberoamericana.

Meneses Morales, E. (I998b). Tendencias educativas oficiales en México, I9II-I934. México: Centro de Estudios Educativos / Universidad Iberoamericana.

Meneses Morales, E. (1998c). Tendencias educativas oficiales en México, 1934-I964. México: Centro de Estudios Educativos / Universidad Iberoamericana.

224 POES. [Periódico Oficial del Estado de Sinaloa] (I874, 25 de marzo). Culiacán Sinaloa.

POES. (I88I, I3 de agosto). Culiacán Sinaloa.

POES. (I88I, 2I de mayo). Culiacán Sinaloa.

POES. (I885, I de marzo). Culiacán Sinaloa.

POES. (I885, 7 de marzo). Culiacán Sinaloa. 
POES. (I916, 27 de enero). Culiacán Sinaloa.

POES. (I9I8, I de junio). Culiacán Sinaloa.

POES. (I922, I9 de diciembre). Culiacán Sinaloa.

POES. (I922, 26 de octubre). Culiacán Sinaloa.

POES. (I93I, 23 de octubre). Culiacán Sinaloa.

POES. (I937, 27 de febrero). Culiacán Sinaloa.

POES. (I94I, I8 de octubre). Culiacán Sinaloa.

Posner, G.J. (1998). Análisis del currículo. Colombia: Ed. Mac Graw-Hill. Rosales Medrano, M.Á. (2009). La formación del profesional del docente de primaria. México: Universidad Pedagógica Nacional / Plaza y Valdez Editores.

Sánchez Gastélum, J.L. (2000). Sociedad y educación en Sinaloa. I874I9I8: El Colegio Rosales [Tesis de Doctorado]. Universidad Autónoma de Sinaloa y Universidad Autónoma de Aguascalientes, México.

\section{Notas}

${ }^{\text {I }}$ Con el nombre de institución rosalina nos referimos a la actual Universidad Autónoma de Sinaloa y sus antecedentes: Liceo Rosales (I873-I874), Colegio Rosales (I874-I9I8), Universidad de Occidente (I9I8-I922), Colegio Civil Rosales (I922-I937), Universidad Socialista del Noroeste (I937-I94I) y Universidad de Sinaloa (I94I-I965). El adjetivo "rosalina" es en reconocimiento al general sinaloense Antonio Rosales, un héroe de la Guerra de la Intervención Francesa quien venció al ejército invasor el 22 de diciembre de I864.

${ }^{2}$ A nivel estatal, esta carrera nació formalmente con la Ley Orgánica de Educación del Estado de Sinaloa (Decreto número 55) aprobada el I8 de enero de 1945 durante el gobierno de Pablo Macías Valenzuela (Beltrán, 20I5b). 
Este artículo se publica bajo una licencia de Creative Commons Reconocimiento-NoComercial 4.0 Internacional, y puede ser usados gratuitamente para fines no comerciales, dando los créditos a los autores y a la revista.

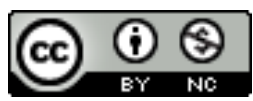

